\title{
How shall we design the future vehicle for Chinese market
}

\author{
Fang Chen*, MinJuan Wang ${ }^{\mathrm{a}}$, Xi Chan Zhu ${ }^{\mathrm{b}}$, Jiaqi $\mathrm{Li}^{\mathrm{b}}$ \\ ${ }^{a}$ Interaction Design, Institute of Apply IT, Chalmers University, 41296 Chalmers, Göteborg, Swedens \\ ${ }^{\mathrm{b}}$ Safety Research, Institute of Automobile, Tongji University, 4800 Cao An Highway,Shanghai, China
}

\begin{abstract}
Surface transportation system is developing very fast in China and the number of vehicles is increasing quickly as well. This development creates a lot of problems on traffic safety and the number of accident is also increasing. In this paper, we made deep analysis of different possible causes of safety problems through three aspects: the traffic environment and infrastructure, in-vehicle information system design and the characteristics of drivers. There are many factors in each aspects may contribute to the transportation safety problems. Problems with infrastructure design and traffic design contribute over $50 \%$ of the traffic accident. Another important factor is that people has very little traffic safety concept and very weak on understanding the important of right behavior on the road. This paper has pointed the urgent needs to study the human factors in road and transportation system and vehicle HMI design, as there are very few such studies available in literature based on Chinese situation. The paper also proposed the needs to develop proactive educational system that can promote driver's understanding of traffic safety and to take the right action during drive.
\end{abstract}

Keywords: Active safety, culture, Chinese market, Chinese driver, HMI, vehicle, traffic

${ }^{*}$ Corresponding author. E-mail: fanch@chalmers.se. 


\section{Introduction}

Soon, China is going to be one of the biggest automobile markets in the world. More and more automobile companies realized that it needs adaptive design of the vehicle for Chinese user, especially when regarding in-vehicle information system interface design and HMI (human-machine interaction) design for ADAS (Advanced Driving Assistance System) systems.

In recent years, the ADAS have a great success in the European market. Most of the new models of cars have equipped with some of the systems like: EBD (Electronic Brake-force Distribution); EBA (Electronic Brake Assist); ASR (Acceleration Slip Regulation); ESP (Electronic Stability Program); ACC (Adaptive Cruise Control system); EBA (Electronic Brake Assistance); lane-departure warning (LDW); forward collision warning (FCW), Blind Spot Detection (BSD), curve management system, city assistant system, night vision, etc. In China, even though the number of vehicles is increasing dramatically, there are still very few cars that are equipped with ADAS system, especially with the systems that require interaction with drivers, such as ACC, FCW, BSD, night vision, etc. This may due to the differences of traffic situation and driver behavior in China compared with Europe.

Aggressive driving made a significant contribution to traffic accident [1]. Statistic shows $84.6 \%$ traffic accidents are caused by vehicle violations [2]. Many factors contribute to the traffic accidents: infrastructural issues, such as the roads were constructed in the wrong way; poorly designed road signs or even lack of them [3]. Many studies indicate that driving behavior is considered as a major safety problem. In 2008 , the report of traffic police office in one province (there are about 33 provinces in China) showed that bad driving habits; poor understanding of other road users and poor respect to traffic regulations were the obvious problems that contribute to the traffic accidents [4].

Lee [5] claims that culture has a significant influence on driving behavior, and it plays a critical role in general driving safety. Lindgren [6] points out that a system is considered useful in one country can be seen as almost worthless (or even harmful) in another one, and system settings feasible in one part of the world may not be suitable on the other side of the globe. Another study [7] indicated the different attitudes towards several types of ADAS systems among Swedish, American, and Chinese groups. Thus it is necessary to take traffic situation, as well as culture factors into consideration when designing the HMI of ADAS for Chinese market.

In the past few years, we have conducted a series studies related to accident analysis, driver behavior, and the culture differences related to HMI design of ADAS systems in China. The comparison between Chinese driver and Swedish drivers were the focus. The studies include: many accidental site visits, interviewing with accident victims, over thousands hours real-time driving records, questionnaires investigation, interviews, observations and laboratory simulation studies, etc. Some of the results have been published in different context on journals and conference proceedings. Some of the data haven't been published yet due to different confidential reasons. In this paper, we do not intend to present a lot of statistic data, rather, provide an in-depth discussion about Chinese traffic situation, the driver behavior, and discuss about how shall we go for next step: what aspects need to be taken into consideration; what areas we need to focus on HMI design for the future vehicles that will be driven in China.

\section{The Traffic Situation in China}

\subsection{The problems with road infrastructure}

China is one of the countries that have made fast development of its surface transportation in the past 30 years. By the end of 2010, China has over 4 million kilometers highway cover the whole country ${ }^{1}$. Only in between year 2009 to 2010 , the road has increased 147 thousand kilometers. The types of road were divided into different categories according to the "owners" of the road: national road, roads belong to the local province, or local counties, or even local villages. Among them, there are over 74 thousand kilometer motorways, which is the second longest in the world. It is interesting to notice that in year 1980, there was no any motorway at all in the whole country! Besides, there are over 3,5 million kilometer roads are located in the countryside.

What does these figures mean? It means that it took over hundred years to develop the surface traffic system in Western countries took less than 30 years in China. This also implies that there are a lot of problems hidden behind the traffic infrastructure system that may contribute to the increased traffic acci-

\footnotetext{
$12011-2015$ 年中国公路行业投资分析及前景预测报告(共四卷) http://www.ocn.com.cn/reports/2006136gonglu.htm
} 
dents in China. These problems can be discussed from the following aspects: the quality of the road, and design of the traffic and the road sign design.

First of all, the quality of the road is very different from place to place. The quality of the roads in the city, outside city, from county to county, is all depends on who design the road and how much money local authority put into the road construction. In a lot of cases, the road was not designed by experts and constructed with acceptable quality control. Therefore, a lot strange designs appeared in the road and made people difficult to drive on it. A new constructed road could be damaged within a couple of months in use. Normally, there is no any road information indicates about the quality of the road. In the countryside, where the majority of the road was built, the quality of the road can be so bad that the drive experience can be extremely sensitive to the weather changes (see figure 1).

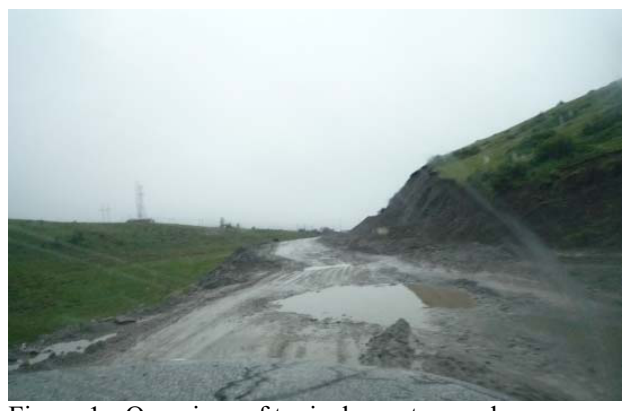

Figure 1 - One piece of typical country road

The picture (figure 1) was taken in a piece of road that is the only major transportation line from one mountain area to another mountain area. As showed in Figure 1, in the raining days, the road is very loblolly. In a sunny day, it can be full with fine dust. In the area where the picture was taken, the local government had once tried to repair the road with concrete surface. After that, there was many more car accidents happened due to the thin ice or water layer on the surface of the road during wintertime and the car drove significantly faster on the road. In the first winter, within less than $50 \mathrm{~km}$ road, seven people were killed! People over speeding and there was not any warning sign about the ice surface and what consequences might be if people drive too fast on icy road! There was no corresponding information neither about what speed people should drive under certain conditions. It may also happen, even the information was provided, people would not obey to it and did as what they used to do.
Second, lack of necessary road sign or the road sign is badly design to be understood. All kinds of mistakes on the road sign design that one could find in the other parts of the world could be found in China. Missing road signs are often happened in the countryside. In the city, it is very often happened that some contradictory road signs are stood side by side. The road sign create confusions to driver. Missing road signs often happened on the countryside roads (see figure 2) as well.

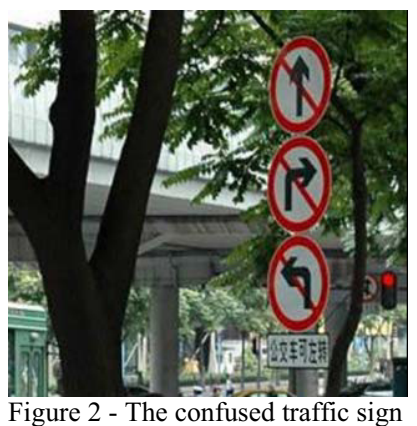

The third, the traffic on the road was designed on the wrong way. One can find such problems almost everywhere. The following traffic design often happened: three lanes suddenly become two lanes after a conjunction without any pre-warning or sign; the lane position was shifted without reasons; one or two lanes suddenly disappeared without any warning sign; the left lane was signed to drive straight, while the right lane beside it was the lane for left turn!

These probably were not the most serious problems compare with the wrong design on the infrastructure. In a lot of cases, the traffic road was designed without taking into account of the driver's behavior and needs. The "usability" of the road is very poor. One typical example is the "Xi Zhi Men motorway bridge in Beijing. It was joked by the drivers as "the $9^{\text {th }}$ miracle in the world", or one of the biggest perplexing maze (See figure 3). People often get lost on this bridge, even with the local citizens. Some drivers said that even they had been drive on this bridge many times, every time, they were not sure about if they took the right turn. Even the traffic policeman does not know how to give instruction to the drivers who gets lost on the bridge. 


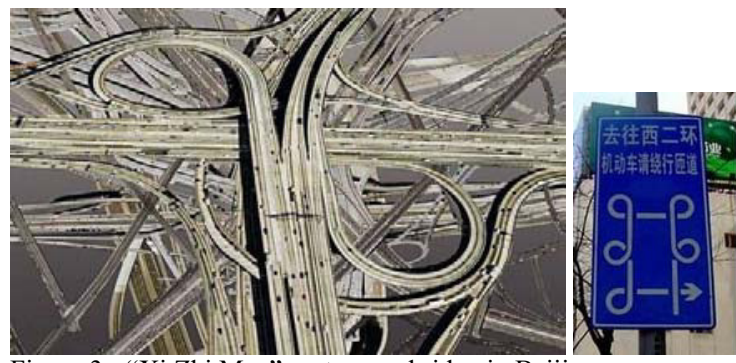

Figure 3 - "Xi Zhi Men" motorway bridge in Beijing

The wrong design of the road without traffic flow indication, or with confused indication may cause a lot of confusions and conflicts in traffic (see figure 4).

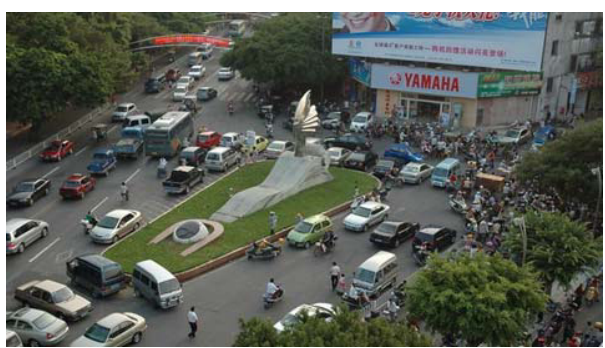

Figure 4 - The confusion of traffic with bad design

All of these problems are probably due to the lack of knowledge on how vehicles are behaving on the road and how other road users interact with each other, and lack of the understanding of traffic safety. All of these indicated that there is an urgent need to carry out the human factor studies on road and traffic in China. The road safety management system is very poor in China as well.

\subsection{The problems with traffic behavior}

There are a lot of reports about the traffic accident in China. It is well known that the accidental rate, and especially the fatality rate at traffic is increasing. It was reported in the 2008 Annual Statistic Report on Road and Traffic Accident in People's Republic of China, that there were 73,484 people killed by traffic accidents, and 304,919 injured. There are also some studies about the cause of these accidents. Xie [1] points out that in China, vehicle violations made a significant contribution to traffic accident involvement. Table 1 showed the statistic result of the major causes of accident in year 2008 in China.
Table 1

The categories of accidents and data

\begin{tabular}{|c|c|c|c|c|c|c|}
\hline \multirow[t]{2}{*}{ Causes } & \multicolumn{2}{|l|}{ cases } & \multicolumn{2}{|c|}{$\begin{array}{l}\text { Fata- } \\
\text { lities }\end{array}$} & \multicolumn{2}{|c|}{$\begin{array}{l}\text { Inju- } \\
\text { ries }\end{array}$} \\
\hline & No & $\%$ & No & $\%$ & No & $\%$ \\
\hline $\begin{array}{l}\text { Not Giv- } \\
\text { ing way }\end{array}$ & 48420 & 18,3 & 9102 & 12,4 & 54769 & 18,0 \\
\hline speeding & 26966 & 10,2 & 10584 & 14,4 & 28793 & 9,4 \\
\hline $\begin{array}{l}\text { Wrong } \\
\text { direction }\end{array}$ & 11959 & 4,5 & 3877 & 5,3 & 16289 & 5,3 \\
\hline $\begin{array}{l}\text { Over } \\
\text { taken }\end{array}$ & 8449 & 3,2 & 2365 & 3,2 & 11011 & 3,6 \\
\hline Drinking & 7518 & 2,8 & 3060 & 4,2 & 7840 & 2,6 \\
\hline $\begin{array}{l}\text { Change } \\
\text { lane }\end{array}$ & 7206 & 2,7 & 1108 & 1,5 & 8804 & 2,9 \\
\hline
\end{tabular}

The data in Table 1 was from national report of the 2008 Annual Statistic Report on Road and Traffic Accident in People's Republic of China. In this report, the accidents were divided into over 47 different causes. In the table, the first cause of accident is "not giving way" when it should. A typical scenario is that in the crossing section, the left turn vehicle should give way to the one that drove straight, but in fact they didn't. This violating behavior cause over $12 \%$ of fatalities and $18 \%$ injuries in traffic accidents. The second is "speeding". It often happened in the evenings or in the countryside. Sometimes, it is not the drivers who were died or injured, but other road users. The third cause was "wrong direction". It means the car drove into the lane that was used for opposite direction driving. This indicated that drivers ignore traffic regulation and drive into wrong directions. This may due to either the traffic sign is not clear, or the driver would like to take a short cut, or to avoid busy traffic, etc. The fourth cause was "over taken". Here it means the car over took another car when it was not in a proper situation. The typical scenarios are that the car over took another car from right side, or when the front car was over taking another car. Drinking and drive is also a very serious problem in China. The sixth biggest cause of accident is due to "change lane" when it was not allowed. It has been noticed by many observations that drivers change lanes too often than necessary during drive, and ignore if it is a solid line or not. In urban areas, there is a higher rate of accidents involves pedestrians, while in suburban districts the higher rate of accidents is correlated with quadricycles. Besides, the rate of accidents involves bicycles and tricycles are also high in both urban and suburban areas [8].

There are many reasons that contribute to the accidental rate in China. We can summarize them here:

First, the high density of traffic and the mix of road users: This is often seen in city traffic. Very 
often, different kinds of road users are mixed in the same parts of traffic. These road users are pedestrians, bicycles, quadricycles, electronic bicycle, cars and different sizes small vehicles and different sizes of trucks.

Second, the concept of traffic safety is very weak. Even though the accidental rate is so high in China, but people still haven't yet build up the necessary concept on traffic safety. Road users did not obey the traffic regulation. Conflictions between different road-users are often seen in every place. Another reason may due to the fact that most private vehicle drivers are the first generation of driver in China. It does not like in Western countries, that people growth up with the cars. Statistic data ${ }^{2}$ showed that in year 1994, there were only 9,4 million cars in total in China. In year 2000, the number increased to 16,1 million. It was 8 to $10 \%$ of increasing every year. In year 2004, the number increased to 27,4 million, which was over $15 \%$ increasing each year. In year 2010 , the number became 90,8 million. It was over $20 \%$ increasing each year. Among this figure, the number of private car owner gets over $30 \%$ increasing only in year 2010 ! This data indicated that there are a lot of un-experienced drivers on the road. This group of people is called "the killer on the road" in China.

Even though, the amount of people who has drive experience is still very few (about $6 \%$ to $10 \%$ ) compare with the large population, who never know how to drive in China. The complicated traffic situation has been developing in recently 20 years. Therefore, most people do not have enough knowledge on traffic safety and have the time to develop a good habit of following traffic regulation.

The large population in China also implies that the education level of the road users and their perception of traffic safety are very different. The fast urbanization development in China turns many villages, and towns into cities and farmers become citizens. This people face a lot of changes in their life. To obey the traffic regulation is difficult because it requires them to change their daily habits and to learn and to remember in their daily activities on the street. One can often hear the comments like "the person drive the car in the way like he rides the bicycle, stop and go on anywhere that is possible for convenient" or "the person walked on the street like promenade in his

\footnotetext{
2 中 0 人民共和国 2010 年国民口和社会口展口公口, 中 人民共和国国家

局, 2011 年 2 月 28 日
}

own backyard, ignored all kinds of traffic signals, vehicles and regulations".

The traffic situation differs between different cities, counties and villages can also make people behave strangely on the street.

\subsection{In vehicle HMI design}

As the traffic situation is much more complicated in China, it would set a high demand on in-vehicle safety and information system design for the vehicles in China. But unfortunately the situation is opposite. At recently, there are a lot of studies on passive safety systems have been carried out in China. Through different public education program and median propaganda, people get to learn the important of using safety belt. There are some studies about drive fatigue, or visual scan pattern. Very few studies regarding the HMI design of in-vehicle information systems based on the culture of Chinese drivers can be found in literature $[3,4,6,7]$.

Almost all kinds of cars that are running in the other parts of the world can be found in China. Some of the Western brand vehicles had some kinds of development teams in China and have their manufactures in China as well. Seldom these teams have the design focus on HMI and safety issues, mostly are on the comfort issues and making the adaptation design. Therefore, the driver interfaces inside Chinese vehicles are similar to those one can found in Western market. There are some cheap cars that are developed by Chinese companies and the HMI design often modified from some other cars without any deep studies behind. There is a misleading idea somehow in China that the advance of technical development is much more important than the HMI design for driver.

Another dangerous factor that may contribute to the increase of the vehicle accidents is the increased tendency of using after-market products, such as mobile phones and GPS, etc.. Again, there is not deep study about how driver may interact with these devices during drive in China. The design of these products hasn't taken the driver safety into consideration.

There are very few cars in China that has equipped with ADAS systems, especially those active safety systems that requires the interaction with drivers. There are a few types of cars that have a few active safety systems with the same technical setting and interface design in Western countries. The only adaptive work has been done in these interfaces is to translate parts of the English texts into Chinese. The 
problem is even more severity. There was no adaptive design and the language is badly translated. As they only translate the parts that were regarded as often used functions, it happens that many functions cannot be used due to language reasons, or do not fit to Chinese driver's mental model and drive situation. The usability of these systems is very low.

It is obviously that HMI design for the vehicles running in China need to fit with Chinese driver's culture background and the traffic situation. The improvement of the interaction design may reduce the number of accidents in China.

\section{The Three Interacted Factors}

There are three main factors that contribute to safety drive (Figure 5). These three factors are closely related to each other.

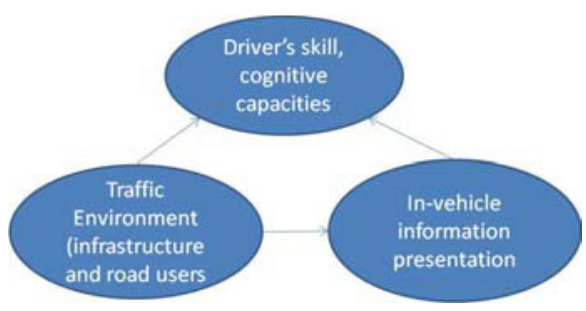

Figure 5 - The factors that affect the safely drive

The factor "traffic environment" includes the road infrastructure design, traffic related information system design and different road users with their behaviors on the road. As we discussed in session 2.1 and 2.2 , there are a lot fundamental problems lying in these categories that need to be taking care of in the first place to be able to reduce the traffic accident in China.

There are a lot of studies related to human factors and traffic safety that has been published in the world (example see [9]). What was missing was the adaptive studies based on Chinese road users' situation. It is not easy to change people's habit in their behavior, but there are a lot of things one can do to change the infrastructure design to avoid the possibility of bad behaviors. The discussion on this issue is out of the scope of this paper.

The factor "in-vehicle information presentation" include both drive related information and entertainment information, as well as information from passive and active safety systems.
The factor "driver's skill, cognitive capacities" includes the driver's skillfulness on driving, his cognitive capacity and limitation, his personality and culture background, etc.

There are a lot of studies that related to the HMI design of passive and active safety system, as well as infotainment system is available in literature and some practical cases. But as Lindgren [3, 4, 6] had pointed out that a good design for one country may not be good for another country, due to the human factor related issues. Therefore, to have a safer drive in China, to protect both car drivers and other road users, the HMI design for both transportation systems and in-vehicle information systems that based on Chinese situation need to be take place urgently. A lot of adaptive studies needs to be carried out based on Chinese traffic, vehicle situation and drivers behavior.

In this paper, we would like to propose the directions on how to approach the necessary HMI design to increase the drive safety in China.

\section{The Approach to HMI Design}

As we discussed in section 3, a lot of human factor and interaction design knowledge in the related field are available based on traffic situations in Western countries. It is urgently needed to carry out the corresponding studies based on Chinese culture and traffic situation. The question is how to approach to the studies? Here we will discuss the question from the three aspects in Figure 5.

\subsection{The understanding of traffic environment}

The traffic environment in China is very different compared with the industrial developed countries. The differences can be considered in many different aspects: the traffic infrastructure design; the high density of traffic; traffic information design and management; the characteristics of different road users and their behavior.

To be able to understand better of the traffic environment and how car drivers interact with other road users, the field operative study can be very interesting. We carried out many thousands of hours video records when the cameras were installed in different vehicles from different cities in China. The analysis of the data is still on the way, and unpublished. From 
recorded 1200 near-crash cases. The top 6 near-crash scenarios (which made up $67 \%$ of total) are:

1. Host car going straight, at a non-junction, the lead vehicle suddenly decelerates (about 26\%)

2. Host going straight, encounters a pedalcyclist at non-junction location (including pedalcyclist drifting, changing to motor lane, stop, deceleration, going opposite direction, left turning) (about 18\%)

3. Host car going straight, at an intersectionrelated location, closes in on a stopped lead vehicle (about 8\%)

4. Host car going straight, at a signalized or nonsignalized intersection-related location, another vehicle making a turning from lateral direction (about $6 \%$ )

5. Opponent vehicle changing lanes/passing object vehicle, encroaches into object's vehicle traveling in the same direction (about 5\%)

6. Host going straight, encounters a pedestrian at a non-junction location (about 4\%)

In the scenario 2, normally the pedalcyclist is the victim. Three causations lead to pedalcyclist riding in the motor vehicle lane: first, non-level road which means the road does not have special lane for nonmotor vehicle (poor road traffic condition), so the pedalcyclist has to drive in motor vehicle lane. Second, the pedalcyclist doesn't obey the traffic rules and drives in motor vehicle lane. Third, the pedalcyclist changes lanes into motor vehicle lane when avoiding the obstacles in non-motor vehicle lane.

In the scenario 6 , pedestrian usually cross the street without walking on the street crossing area.

Many traffic conflicts and recorded near-crash due to the situation of double yellow lights. This is often the case when both yellow lights in two perpendicular directions of intersection are twinkling. Drivers need to pay attention when drive into an intersection with double yellow light.

Normally, people said that over $98 \%$ of traffic accidents are due to the mistakes made by drivers. When we take close look at the accident analysis and near-crash analysis from the record in China, we found that $19 \%$ of accidents are due to the mistakes of the drivers, while $31 \%$ due to the technical problems of the vehicles and $50 \%$ actually cause by the problems in the traffic infrastructure and environment.

This analysis implied that to design the in-vehicle HMI system based on the common understand of traffic regulation would not work in the street in China.

\subsection{Increase the traffic safety awareness for the drivers}

In China, the technical level of personal vehicles on the street differs dramatically, from the most luxury ones in the world to some of the cheapest ones. The luxury ones normally are imported directly from the producers outside China. The cheapest cars are normally easy to encounter with technical problems and very weak against crash.

Whatever the technical level of the car is, the driver's traffic safety concept would be the same. It is obvious that we need to promote the traffic safety concept and encourage the right behavior to the drivers. The question is how. To increase a lot of the costs of the vehicle for a better design is not the best option, because people doesn't yet ready to pay a lot of money for safety. Therefore some simple and cheap solution can have a good market.

Safety education through drive school, through different campaigns in the society, and installing different safety facilities inside the car can be the ways, but some proactive and continuous education devices may provide a good help for people to develop a safety drive habit. Such devices should be developed based on deep understand of driver's needs and wants, the changed traffic situation and driver's behavior.

For example, people do not like to put on the safety belt because normally in city drive, the traffic flow can be very slow and people would feel very uncomfortable to have the safety belt on. When car was stopping, or in a very slow movement, the safety belt may not be so critical, but when the car speed increasing, to put on the safety belt can be very important, but in that moment, people may simply forgot to put it on. Then if we can have a system that can sense the car speed and remind the driver about the safety belt in an interesting way, the driver may put it on.

The continuous education system can also remind the driver the attention to what special issue, such us in bad weather, one should not over speeding; one should put the front lights on when it is dark; remind people not to over taken from right side, etc. It can also sense the drive habit of the driver and indicate the effects from different bad behavior and encourage the good behavior.

For such a system to be accepted by drivers, the emotional issue and pleasurable design of the interaction is extremely important besides the understanding of the driver and traffic situation. Otherwise, people 
would not accept a device that monitoring his behavior and continuously criticize him.

\subsection{HMI design for in-vehicle information systems}

In-vehicle information systems include both driver related information system design and infotainment system design. For the drive related safety system interaction design, the new challenges will be on how to design the interaction for active safety systems. First of all, it is important to identify what kinds of system are the most needed for Chinese drivers. We carried out some questionnaires investigation on this issue and compared the data between Chinese and Swedish. The data showed very different preference of active safety systems between these two countries. The first preferable system for Chinese was the backup monitor, while for Swedish was traffic sign recognition system. The selection of active safety system for Chinese driver should be based on analysis of traffic environment situation to identify the needs, rather than the wants from the drivers. The constraints from traffic environment are very different in China compared with Europe countries.

There is not published statistics about the relationship between accident and the performance of infotainment systems during drive. In China, mobile phone use during drive is not prohibited. Different after-market infotainment products are introduced to drivers, for example DVD players or TV receivers. These systems do not have any "safety guards" that can filter the functions based on different driving

\section{References}

[1] C. Xie and D. Parker, -A social psychological approach to driving violations in two Chinese cities, Transportation Research Part F, vol. 5, pp. 293-308, Jun. 2002.

[2] 占，一注道路安全-第一届中国道路交通安全，世 界汽 , pp. 26-27, May. 2007.

[3] A. Lindgren, F. Chen, P.W. Jordan, and P. Ljungstrand, -Cross-cultural issues and driver requirements for advanced driver assistance systems, in proceedings of the 2nd International Conference on Applied Human Factors and Ergonomics, Las Vegas, US, 2008.

[4] A. Lindgren, F. Chen, P.W. Jordan, and H. Zhang, -Requirements for the design of advanced driver assistance systems - the differences between Swedish and Chinese drivers, International Journal of Design, vol. 2, no. 2, pp. 41-54, Aug. 2008.

[5] J.D. Lee, -Driving safety, In Review of Human Factors, R. S. Nickerson, Ed. Santa Monica, CA: Human Factors and Ergonomics Society, pp. 172-218, 2006. situation. The HMI design for these systems, and coordinate with drive situation need to be studied.

\section{Conclusion}

Surface transportation system is developing very fast in China in recently years and the number of vehicles is increasing quickly. This fast development creates a lot of problems on traffic safety and the number of accident is also increasing. In this paper, we made deep analysis of different possible causes of safety problems through three aspects: the traffic environment and infrastructure, in-vehicle information system design and the characteristics of drivers. There are many factors in each aspects may contribute to the transportation safety problems. One of the important factor is that people has very little traffic safety concept and very weak on understanding the important of following traffic regulations on the road. This paper has pointed the urgent needs to study the human factors in road and transportation system design and vehicle HMI design, as there are very few such studies available in literature. The paper also proposed the needs to develop proactive educational system that can promote driver's understanding of traffic safety and to take the right action during drive.

[6] A. Lindgren, F. Chen, P.W. Jordan, and P. Ljungstrand, -Do you need assistance? - naturalistic case studies investigating the need of advanced driver assistance systems during normal driving, |l, unpublished, Chalmers University of Technology, Sweden, 2007.

[7] A. Lindgren, R. Broström, F. Chen, and P. Bengtsson, - Driver attitudes towards advanced driver assistance system - cultural differences and similarities, $\|$ in Human Factors Issues in Complex System Performance, D. de Waard, G.R.J. Hockey, P. Nickel, and K.A. Brookhuis, Ed. Maastricht, the Netherlands: Shaker Publishing, 2007, pp. 205-215.

[8] 占，“探 我国道路安全 状- 第二 中国道路安全主 ,”世界汽 , pp. 18-19, Jun. 2008.

[9] R. E. Dewer, P. L. Olson, Human Factors in Traffic safety, second edition, by Lawyer and Judges Publishing Company, Inc, 2007. 